\title{
Implementación de un laboratorio de cómputo con software y hardware libres
}

\section{Implementation of a computer lab with free software and hardware}

GARCÍA-ROMO, Héctor Salvadori๋*, ÁVILA-HERNÁNDEZ, José César, ÁVILA-SOTO, Ernesto Alonso y MEZA-GUTIÉRREZ, Amparo Jazmín

Universidad Tecnológica de Bahía de Banderas, Nayarit, México

ID $1^{\text {er }}$ Autor: Héctor Salvador, García-Romo / ORC ID: 0000-0001-5847-6739, CVU CONACYT ID: 903493

ID $1^{\mathrm{er}}$ Coautor: José César, Ávila-Hernández / ORC ID: 0000-0002-4606-358X, CVU CONACYT ID: 301707

ID $2^{\text {do }}$ Coautor: Ernesto Alonso, Ávila-Soto / ORC ID: 0000-0002-4499-5914, CVU CONACYT ID: 737754

ID $3^{\text {er }}$ Coautor: Amparo Jazmín, Meza-Gutiérrez / ORC ID: 0000-0002-1616-9199, CVU CONACYT ID: 446050

\section{Resumen}

Objetivos: Implementar un laboratorio de cómputo de bajo costo, utilizando hardware libre como Raspberry PI y software libre como Linux, para el apoyo de las escuelas primarias que no cuenten con la capacidad económica para solventar la compra de un laboratorio de cómputo convencional, con la finalidad de incrementar el interés de los alumnos de primero a 6to grado del uso de las TIC en la vida diaria. Metodología: Se realizó un análisis de las diferentes distribuciones linux existentes para la raspberry PI, así mismo, se analizaron los diferentes programas de software libre que serían utilizados en las aulas, como matemáticas, historia, lectura, ciencias y principios de programación. Contribución: El uso de herramientas de software libre en el aula, no solo es cuestión de economía, si no que también la enseñanza de las 4 libertades del software libre, utilizar, compartir, distribuir, y la posibilidad de mejorarlo conociendo el código fuente, además promueve el trabajo colaborativo, y la utilización del hardware libre tiene como ventaja, la independencia tecnológica.

Software Libre, Hardware Libre, Raspberry PI

\begin{abstract}
Objetives: To implement a low cost computer laboratory, using free hardware such as Raspberry PI and free software such as Linux, for the support of primary schools that do not have the economic capacity to solve the purchase of a conventional computer laboratory, in order to increase the interest of students from first to 6th grade of the use of ICT in daily life. Methodology: An analysis of the different existing linux distributions for the raspberry PI was carried out, as well as the different free software programs that would be used in the classrooms, such as mathematics, history, reading, science and programming principles. Contribution: The use of free software tools in the classroom, is not only a matter of economy, but also the teaching of the 4 freedoms of free software, use, share, distribute, and the possibility of improving it knowing the source code. It also promotes collaborative work, and the use of free hardware has the advantage of technological independence.
\end{abstract}

Free Software, Free Hardware, Raspberry PI

Citación: GARCÍA-ROMO, Héctor Salvador, ÁVILA-HERNÁNDEZ, José César, ÁVILA-SOTO, Ernesto Alonso y MEZA-GUTIÉRREZ, Amparo Jazmín. Implementación de un laboratorio de cómputo con software y hardware libres. Revista de Simulación Computacional. 2019. 3-9: 27-33

\footnotetext{
* Correspondencia al Autor: (hgarciar@utbb.edu.mx)

$\dagger$ Investigador contribuyendo como primer autor.
} 


\section{Introducción}

En la actualidad, el uso de una computadora en el salón de clase se convierte más que en un lujo, en una necesidad, lamentablemente las escuelas públicas de nivel primaria no cuentan con los recursos para la implementación de laboratorios de cómputo costos, y en muchos de los casos los alumnos no cuentan con computadoras en casa. El presente trabajo da a conocer la utilización de hardware libre como la Raspberry Pi, y Sistemas Operativos Libres y software educativo libre, como una alternativa viable para montar un laboratorio de cómputo con pocos recursos financieros, pero con un alto impacto en el aprendizaje de los alumnos, ya que tendrán la oportunidad de utilizar dicha tecnología que de otro modo sería muy difícil que lo pudieran lograr (Q4OS, 2019) (KANO, 2019).

Se realiza un análisis de los diferentes sistemas operativos libres en el mercado, sus principales características para así,determinar cuál se adapta de mejor manera al hardware de la RaspBerry Pi, posterior a ello, se realiza un análisis del software libre que debe de tener precargado el sistema operativo que cubra con los requerimientos de las asignaturas que se imparten desde primero a sexto grado de primaria.

\section{Análisis del Sistema Operativo Libre}

En el mercado de los sistemas operativos de distribución libre, existe una gran variedad de alternativas que se adaptan de manera adecuada a la Raspberry Pi, los más destacados son los de la Tabla 1, dichos sistemas contienen características esenciales para trabajar con este proyecto. Se eligieron por ser distribución libre y ser parte de la familia Linux y por su compatibilidad con la tecnología Raspberry pi. Cada sistema operativo tiene características que lo diferencian de otros, pero todos comparten características similares como que son de distribución libre, además, existen comunidades de desarrolladores que dan soporte a dichos proyectos, esto es de suma importancia ya que se requiere que exista un respaldo técnico del sistema elegido para el proyecto.

\begin{tabular}{|c|l|c|l|}
\hline$\#$ & Sistema Operativo & $\#$ & Sistema Operativo \\
\hline 1 & Ubuntu Core & 6 & Q4OS \\
\hline 2 & Doudoulinux & 7 & OSMC \\
\hline 3 & Kano Os & 8 & Risc OS \\
\hline 4 & Qimo 4 Kids & 9 & Ubuntu Mate \\
\hline 5 & Linux KidX & 10 & Raspbian \\
\hline
\end{tabular}

Tabla 1 Sistemas Operativos de distribución libre
De acuerdo a las características de los mismos se redujo la lista a los siguientes 5 sistemas operativos:

$\begin{array}{ll}- & \text { Ubuntu Mate } \\ - & \text { Q4OS } \\ - & \text { Raspbian } \\ - & \text { Kano OS } \\ - & \text { Quimo 4 Kids }\end{array}$

Dichos sistemas se eligieron por las siguientes razones:

Ubuntu Mate Todos los sistemas operativos Ubuntu cuentan con las mismas características básicas, Ubuntu Mate ofrece una sencillez en su interfaz gráfica, además que su rendimiento lo hace ideal en este tipo de proyecto, además, está mantenida por la comunidad y es un derivado de Ubuntu oficialmente reconocido por la empresa Canonical, Ltd., por lo que no se tienen problemas de que vaya a ser un proyecto olvidado.

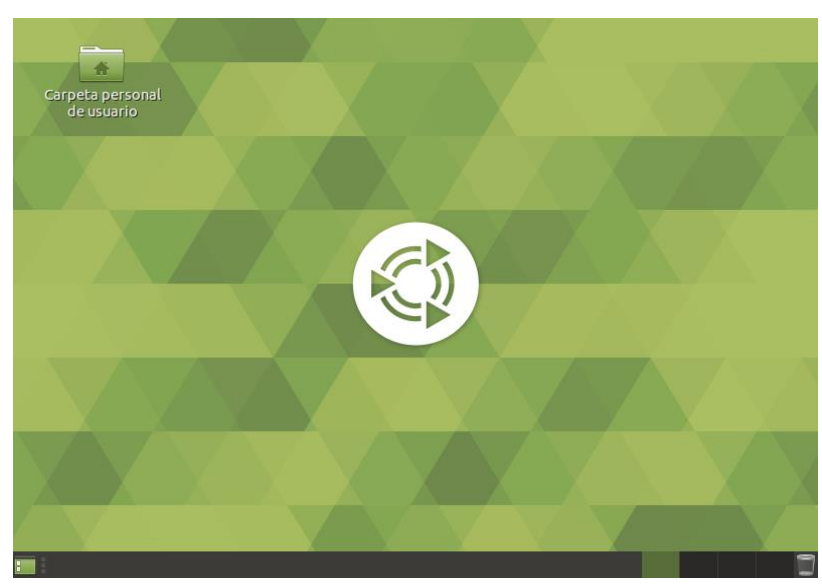

Figura 1 Interfáz Ubuntu Mate

Q40S Ofrece una interfaz ligera y amigable con el usuario novato. Tiene un enfoque orientado específicamente a usuarios familiarizados con Windows permite también reutilizar aquellas antiguas computadoras, abandonadas en algún lugar por limitaciones de hardware. Cuenta con soporte para efectos y ofrece dos apariencias visuales diferentes uno similar a Windows XP y el otro similar a Windows 7, dentro de las aplicaciones que podemos encontrar están Firefox, Google Chrome, LibreOffice, VLC, Thunderbird, Synaptic, Shotwell y Konqueror. 


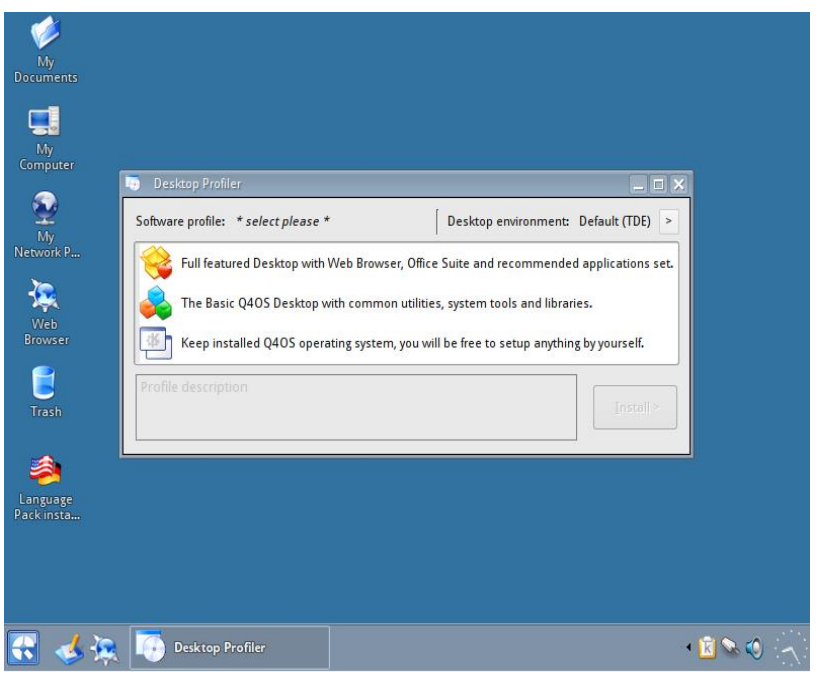

Figura 2 Interfaz Q4OS

Raspbian. La distribución es ligera para moverse ágilmente en el hardware de la Raspberry $\mathrm{Pi}$, con un entorno de escritorio LXDE y Midori como navegador web predeterminado. Además, incluye herramientas de desarrollo muy interesantes, como IDLE para Python, Scratch para programar videojuegos (muy interesante sobre todo si se combina con Arduino), la tienda de aplicaciones denominada Pi Store, etc. Programas por defecto:

\section{- $\quad$ LibreOffice, Navegador Web \\ - Diferentes herramientas de programación (Python \\ - Calculadora, Visor de imágenes, PDF, Editor de texto,etc.}

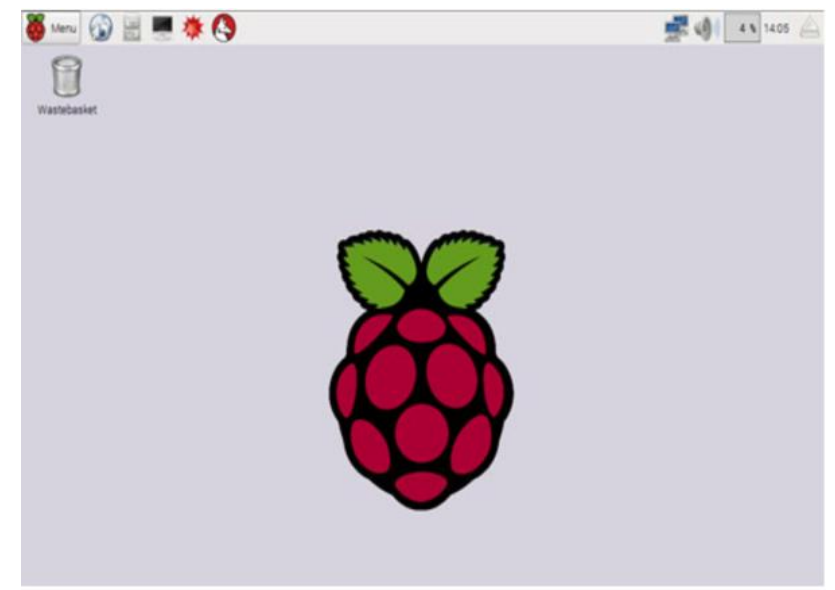

Figura 1 Interfáz Raspbian
Kano OS Tiene un diseño muy simple y es muy fácil de usar. En ella se encuentran juegos, aplicaciones de vídeo y de música, herramientas para aprender a programar y otras utilidades dirigidas a los más jóvenes. En tal sentido, las características de Kano OS, son bastante interesantes, estas sirven exclusivamente para fomentar el interés por el mundo de la tecnología y la programación desde la edad inicial, además permite ir aprendiendo las bases de un lenguaje conocido como Python o Javascript, con el cual se puede ir arrastrando y soltando bloques, mientras se puede observar que el código de tiempo, juegos y canciones, se pueden cambiar en tiempo real, así como también las aplicaciones.

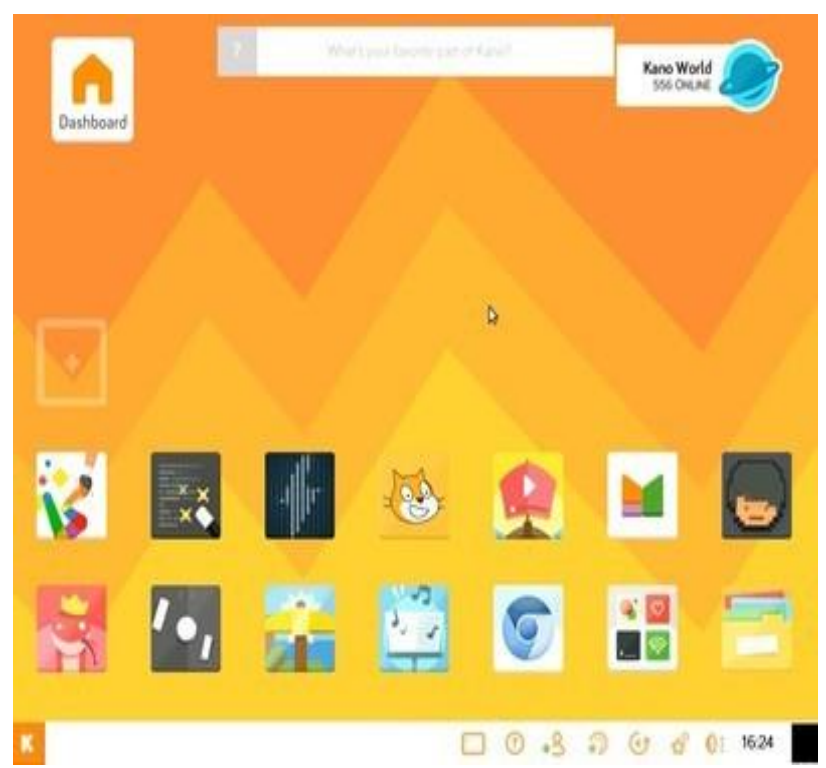

Figura 2 Interfáz Kano OS

Qimo 4 Kids diseñada para los más pequeños, este sistema se especializa en ofrecer aplicaciones para niños y así puedan expandir sus conocimientos en matemáticas, español, geografía y otras áreas. Lo primero que llama la atención de Qimo es su intuitiva interfaz, que cuenta con el gestor de ventanas XFCE.

En la parte inferior, una barra con iconos nos permitirá ejecutar distintos programas, la mayor parte educativos, ya que uno de los objetivos de esta distribución es hacer pasar un buen rato a los más pequeños al mismo tiempo que aprenden. Entre las aplicaciones incluidas, destaca GCompris, una colección de juegos de todo tipo, apta a partir de los 2 años, aunque podremos disfrutar de otras como Childsplay o Etoys, entre otros. 


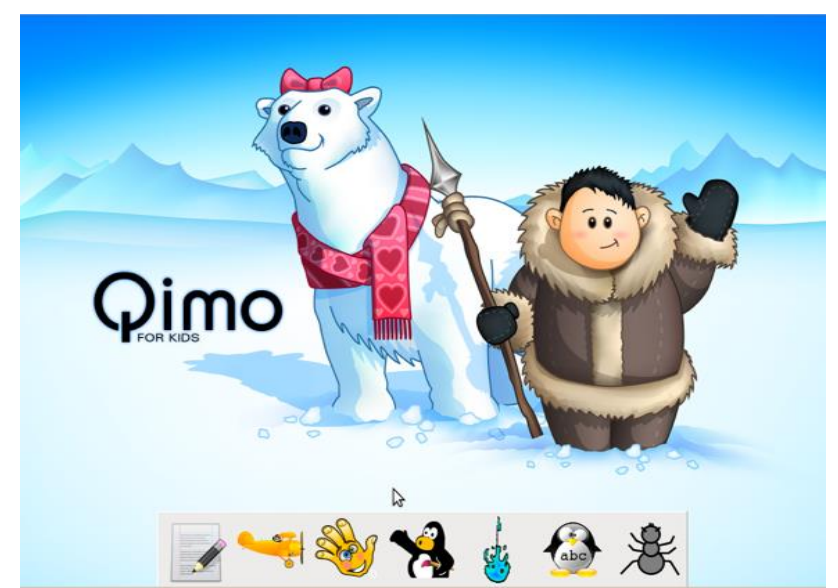

Figura 3 Interfáz Quimo

\section{Análisis del Software Educativo Libre}

Las grandes ventajas que tiene el software libre han hecho que tenga gran popularidad en el ámbito educativo, tanto estudiantes como docentes de diferentes niveles educativos utilizan una amplia gama de aplicaciones que fomentan el aprendizaje sin costo alguno.

Para la eduación primaria existe un alto número de programas que facilitan el aprendizaje básico, como mátemáticas, ciencias y Geografía entre otros, a continuación se describen algunos programas que serían de gran utilidad en el aula.

\section{GCompris}

Es un conjunto de programas educativos de alta calidad que contiene un gran número de actividades para niños entre 2 y 10 años.

Algunas actividades están orientadas a juegos, pero siguen siendo educativas.

Esta es una lista de categorías de actividades con algunos ejemplos:

\section{Descubriendo la computadora:} teclado, ratón, pantalla táctil...

Lectura: letras, palabras, práctica de lectura, escritura de texto...

Aritmética: números, operaciones, memoria en tablas, enumeración, tabla de doble entrada...

Ciencia: esclusa de canal, el ciclo del agua, energía renovable...
Geografía: países, regiones, cultura...

Juegos: ajedrez, memoria, alinear 4, juego del ahorcado, tres en raya...

Otros: colores, formas, alfabeto Braille, aprender a decir la hora...

En la actualidad, GCompris ofrece más de 100 actividades y hay más en desarrollo. GCompris es software libre, por lo que tiene la posibilidad de adaptarlo a sus necesidades, de mejorarlo y, lo más importante, de compartirlo con niños de todo el mundo. El proyecto GCompris está patrocinado y desarrollado por la comunidad KDE.

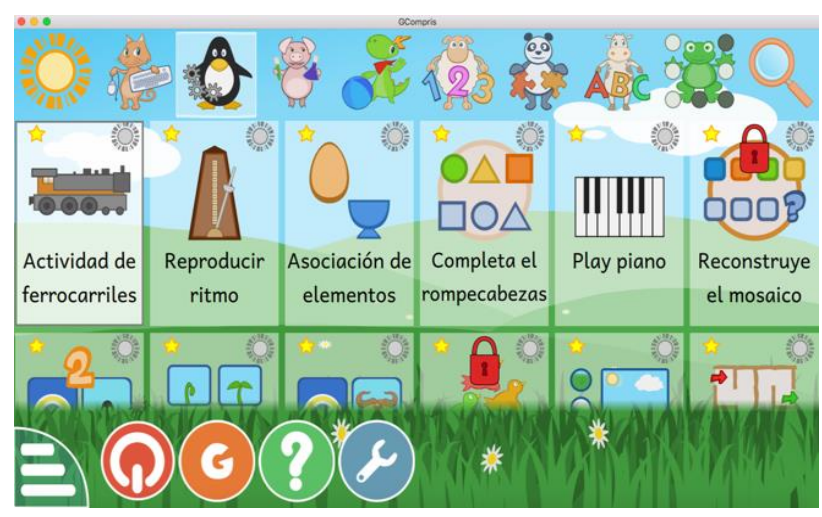

Imágen 4 Programa Educativo GCompris

\section{Tuxmath}

Es un juego de acción para aprender matemáticas y agilizar el cálculo mental. En el juego, un simpático pingüino, armado con un rayo láser, trata de proteger a sus iglúes de las operaciones matemáticas. Cada vez que resuelve una operación, evita la destrucción de un iglú. El juego, que es similar a otros programas muy populares entre niños y niñas, permite desarrollar el cálculo mental de una forma entretenida. Es un entretenido juego educativo en el que la agilidad mental realizando restas, sumas, multiplicaciones y divisiones, así como la velocidad para introducir el resultado y disparar serán las claves para salvar al planeta.

Entre las características de este singular juego tenemos:

Soporte para múltiples usuarios (útil para las escuelas)

Tutoriales en pantalla

Tabla de puntuaciones máximas

Modo de formación - más de 50 clases de paquetes. 
- $\quad$ Soporte multiplataforma ( Linux, Windows, Mac OS X, BeOS )

La localización de más de treinta idiomas.

Soporta el modo multijugador inalámbrica a internet.

Misiones

- $\quad$ Factoroids: Clon del clásico de Atari de los videojuegos "Asteroids", modificado para que sea una actividad para capacitar a la factorización.

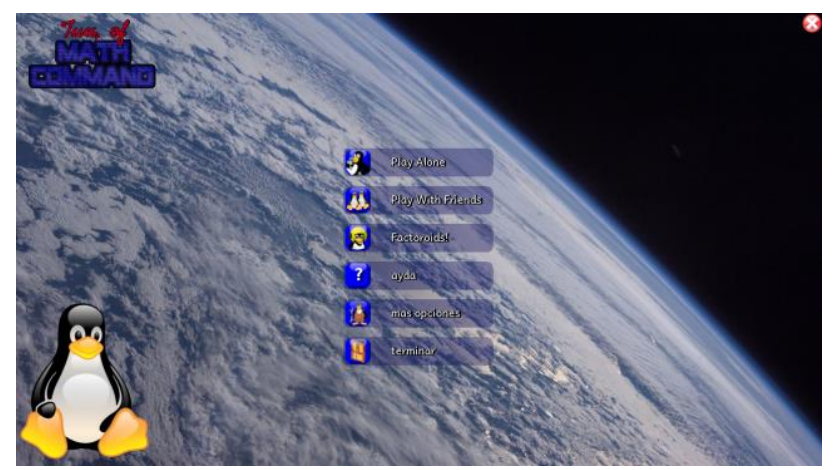

Figura 5 Pantalla principal de Tuxmath

Como se puede observar en la Figura 8, en la academia de entrenamiento de matemáticas, para poder pasar las misiones hay que realizar operaciones matemáticas como sumas y restas.

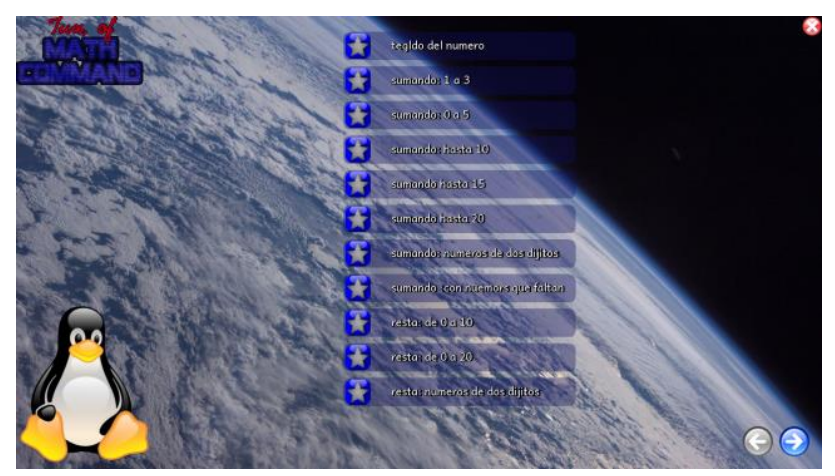

Figura 6 Academia de entrenamiento de Matemáticas

\section{Klettres}

Esta aplicación es ideal para niños que estén aprendiendo a reconocer las letras o sílabas. También es de gran utilidad para todos aquellos usuarios que estén aprendiendo un nuevo idioma, dado que se encuentra hasta en más de 30 lenguas. Desarrollo de Secciones y Apartados del Artículo con numeración subsecuente

El programa elige una letra o una sílaba de forma aleatoria, esta letra o sílaba se muestra en pantalla y se escucha su sonido.
El usuario debe entonces escribir esta letra o sílaba. El entrenamiento finaliza en los niveles en los que no se muestra ninguna letra o sílaba, y simplemente se escucha su sonido. No es necesario que el usuario sepa manejar el ratón, basta con utilizar el teclado.

En este momento están disponibles veinticinco idiomas, si el idioma de su KDE está entre ellos, entonces vendrá de forma predeterminada; de no ser así, el idioma predeterminado será el francés. Puede obtener fácilmente cualquier idioma adicional usando el menú Archivo y Obtener alfabeto en un nuevo idioma..., siempre que su equipo esté conectado a Internet.

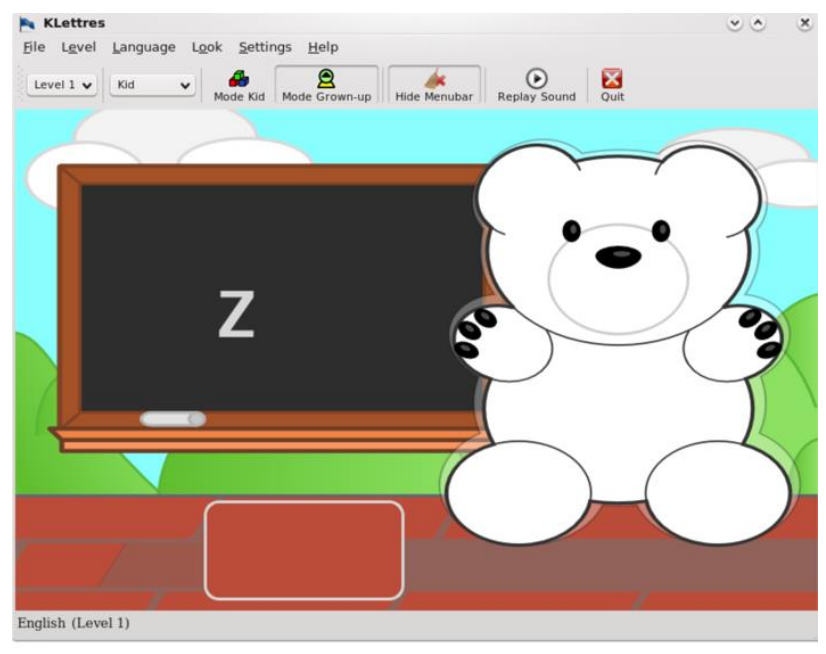

Figura 7 Kletrees interfaz Kids

Existen dos modos diferentes que le permiten adaptar KLettres a sus necesidades y tener la habitual interfaz completa o una interfaz reducida. Un niño no verá la barra de menú. Por tanto se asume que un niño no va a querer cambiar el idioma por sí mismo. Una lista desplegable le permitirá elegir entre los diferentes niveles. Para un usuario adulto, en cambio, el fondo no será tan infantil y la barra de menú estará presente. En los tres temas existentes (Infantil, Desierto y Sabana) existirán diferentes fondos y tipos de letra. 


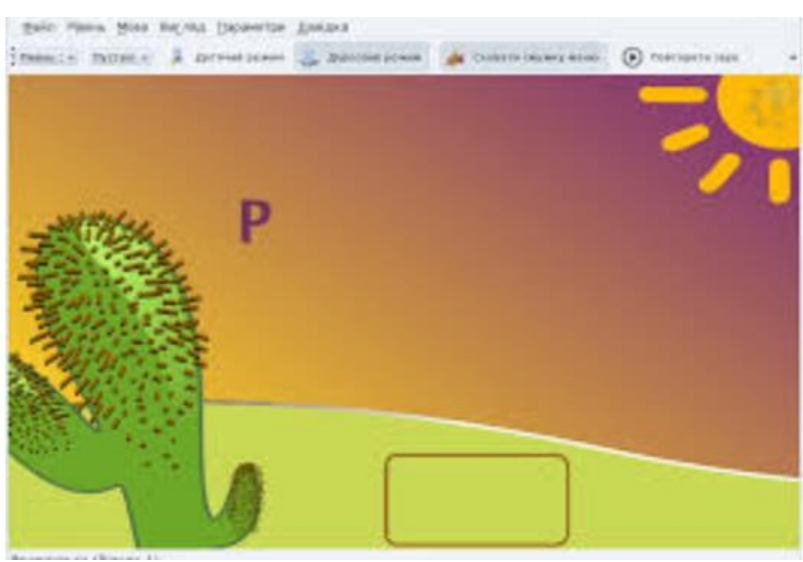

Figura 8 Kletrees Interfaz Desierto

\section{Geogebra}

GeoGebra es un software de matemáticas para todo nivel educativo. Reúne dinámicamente geometría, álgebra, estadística y cálculo en registros gráficos, de análisis y de organización en hojas de cálculo. GeoGebra, con su libre agilidad de uso, congrega a una comunidad vital y en crecimiento. En todo el mundo, millones de entusiastas lo adoptan y comparten diseños y aplicaciones de GeoGebra. Dinamiza el estudio.

Armonizando lo experimental y lo conceptual para experimentar una organización didáctica y disciplinar que cruza matemática, ciencias, ingeniería y tecnología (STEM: Science Technology Engineering \& Mathematics). La comunidad que congrega lo extiende como recurso mundial, potente e innovador para la cuestión clave y clásica de la enseñanza y el aprendizaje.

\section{GeoGebra reúne gráfica y} dinámicamente álgebra y geometría, análisis y hojas de cálculo.

Potentes herramientas en armonía con una interfaz intuitiva y ágil

Herramienta de autoría para crear recursos de aprendizaje interactivos como páginas web ¡Políglota! Porque está disponible en cada idioma requerido por los millones de usuarios del mundo.

Software de código abierto libre y disponible para usos no comerciales.

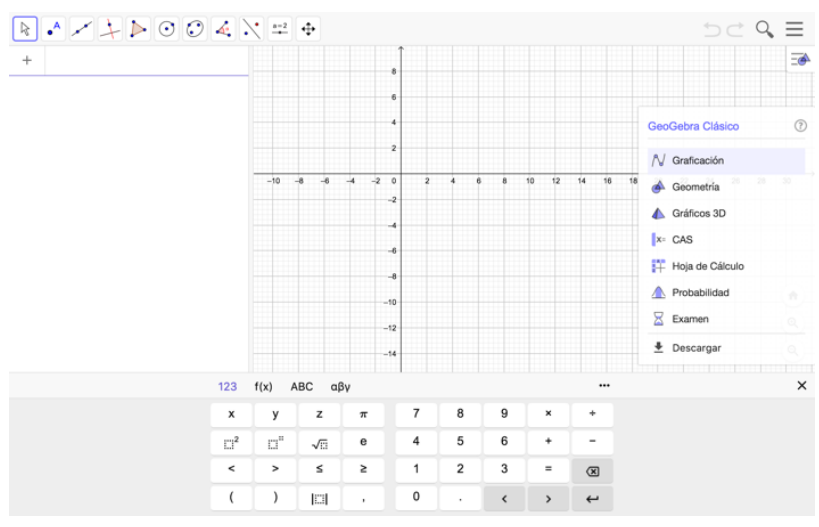

Figura 9 Interfaz GeoGebra

\section{Scratch}

La habilidad para crear programas de ordenador es una parte importante de la alfabetización en la sociedad actual. Cuando la gente aprende a programar en Scratch, aprende importantes estrategias para resolver problemas, diseñar proyectos y comunicar ideas.

Scratch está diseñado especialmente para edades entre los 8 y 16 años, pero lo usan personas de todas las edades. Con Scratch puedes programar tus propias historias interactivas, juegos y animaciones y compartir tus creaciones con otros en la comunidad online.

Scratch ayuda a los jóvenes en su aprendizaje y a pensar de forma creativa, a razonar sistemáticamente, y a trabajar de forma colaborativa - habilidades esenciales para la vida en el siglo 21.

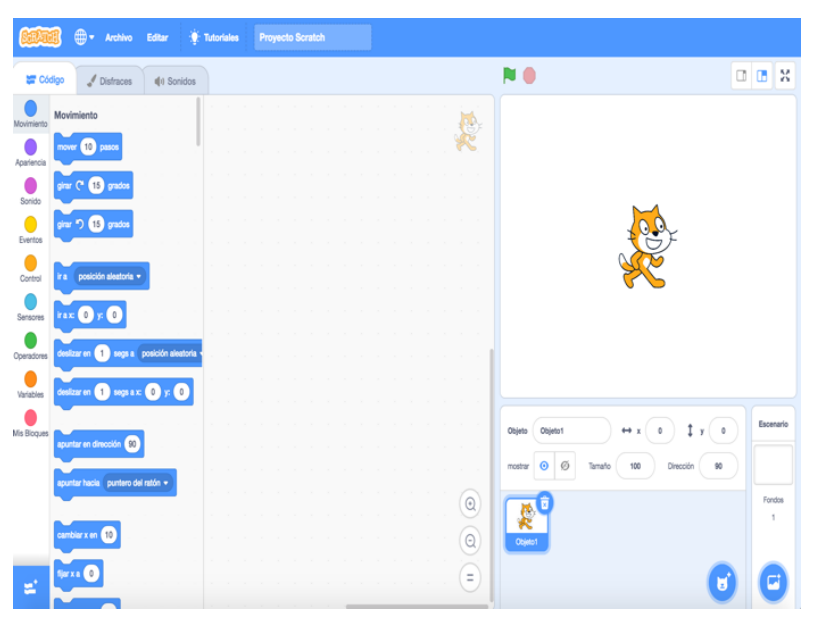

Figura 10 Interfáz Scratch 


\section{Metodología a desarrollar}

Para poder seleccionar las mejores alternativas de sistemas Operativos Libres se realizó la descarga de cada uno de los ISO, y se realizó la instalación de cada uno de ellos en una memoria microsd para poder realizar las pruebas pertinentes en la respberry pi b, el software libre educativo, se descargó directamente sobre los dos sistemas operativos elegidos, Quimo4Kids y Raspbian y se instalaron y se realizaron las pruebas.

\section{Resultados}

En lo que respecta a los resultados del Sistema Operativo, se concluye que los 5 analizados cubren con las especificaciones del Hardware de una Raspberry pi B, y dada la facilidad de instalación en una memoria micro SD, se opta por tener instalado Quimo4Kids para los primeros tres años de primaria, contando como principal software el Gcompris y Raspbian para los grupos de cuarto a sexto de primaria.

\section{Conclusiones}

Existe una gran cantidad de software libre educativo, de entre todas las opciones se seleccionaron los 5 programas que más aportan a la enseñanza a nivel primaria en lo que respecta a la enseñanza de las matemáticas, el algebra, geometria, así como el uso de vocabulario, música y principios básicos de programación entre otros, pudiendo incorporar software extra de ser necesario y con la ventaja que es software libre y gratuito.

Por otra parte se cuenta con la ventaja que todos los programas antes mencionados, pueden ser instalados en cualquiera de los dos sistemas operativos seleccionados.

\section{Referencias}

Lifelong Kindergarten . (2019). Scratch. Obtenido de https://scratch.mit.edu/

Canonical Ltd. (2019). Ubuntu Mate. Obtenido de https://ubuntu-mate.org/download/

Ecured. (2019). Enciclopedia colaborativa en la red cubana. Obtenido de TuxMath: https://www.ecured.cu/TuxMath

GeoGebra. (2019). GeoGebra. Obtenido de https://www.geogebra.org/
Hall, M. (2019). Quimo for Kids. Obtenido de http://www.qimo4kids.com/download/

KANO. (2019). KANO OS. Obtenido de https://kano.me/downloadable/eu

Kde.org. (20 de Abril de 2016). Kde. Obtenido de

https://docs.kde.org/stable5/es/kdeedu/klettres/i ndex.html

Q4OS. (2019). Q4OS. Obtenido de https://q4os.org/downloads1.html

Raspbian.org. (2019). Raspbian. Obtenido de https://www.raspbian.org

Timothée Giet and others. (11 de Agosto de 2019). Gcompris. Obtenido de https://gcompris.net/index-es.html 